\title{
Can We Verify an Elephant?
}

\author{
David Harel \\ Weizmann Institute of Science
}

\begin{abstract}
The talk shows the way techniques from computer science and software engineering can be applied beneficially to research in the life sciences. We will discuss the idea of comprehensive and realistic modeling of biological systems, where we try to understand and analyze an entire system in detail, utilizing in the modeling effort all that is known about it. I will address the motivation for such modeling and the philosophy underlying the techniques for carrying it out, as well as the crucial "verification" question of when such models are to be deemed valid, or complete. The examples I will present will be from among the biological modeling efforts my group has been involved in: $\mathrm{T}$ cell development in the thymus, lymph node behavior, organogenesis of the pancreas, fate determination in the reproductive system of C. elegans, and a generic cell model. The ultimate long-term "grand challenge" is to produce an interactive, dynamic, computerized model of an entire multi-cellular organism, such as the C. elegans nematode worm, which is complex, but well-defined in terms of anatomy and genetics. The challenge is to construct a full, true-to-all-known-facts, 4-dimensional, fully animated model of the development and behavior of this worm (or of a comparable multi-cellular animal), which is easily extendable as new biological facts are discovered.
\end{abstract}

